\title{
List of Reviewers 2015
}

The Journal of Pediatric Rehabilitation Medicine would like to recognize the following reviewers for their time and commitment in providing scientific peer reviews in the past year.

\begin{tabular}{|c|c|}
\hline Pasquale Accardo & Christine Kaufman \\
\hline Rishi Agrawal & Sudha Kaul \\
\hline Susan Apkon & Howard King \\
\hline Joan Arvedson & Brad Kurowski \\
\hline Joann Bodurtha & Susan Mahan \\
\hline Paula Bray & Miriam Manary \\
\hline Timothy Brei & Warren Marks \\
\hline Diane Bryen & Amy McPherson \\
\hline Marco Castori & Linda Michaud \\
\hline James Chinarian & Freeman Miller \\
\hline Karen Choong & Eugenio Monasterio \\
\hline Robert Cohn & Nancy Murphy \\
\hline Robin Cotton & Virginia Nelson \\
\hline Diane Damiano & Michael Nigro \\
\hline Thomas Davis & Sharon O'Neil \\
\hline Kathryn Davitt & Mark Peterson \\
\hline Marcel Dijkers & Frank Pidcock \\
\hline Rochelle Dy & Peter Rosenbaum \\
\hline Jorina Elbers & Richard Segal \\
\hline Ellen Fung & Parag Shah \\
\hline Steven Geiringer & David Sherry \\
\hline Wendy Goodwin & Lotta Sjögreen \\
\hline Mark Gormley & Beth Slomine \\
\hline Michael Green & Virginia Stallings \\
\hline Lisa Griffin & Samanmali Sumanasena \\
\hline Juliet Haarbauer-Krupa & Stacy Suskauer \\
\hline Amy Houtrow & Deborah Thorpe \\
\hline Andrew Hull & Rickey Tivis \\
\hline Edward Hurvitz & Jilda Vargus-Adams \\
\hline Susan Iannaccone & Laura Vogtle \\
\hline Michael Iv & Kate Wan-Chu Chang \\
\hline Michelle Jackman & Seth Warschausky \\
\hline Denise Justice & Robert Watson Scott \\
\hline Nadja Kadom & Rachel Westman \\
\hline
\end{tabular}

